\title{
CARACTERIZAÇÃO DA FARINHA DA CASCA DE DIFERENTES CULTIVARES DE MANGA
}

\author{
Ana Cecilia Poloni Rybka ${ }^{1}$, Arlete de Souza Lima², Rita de Cássia Mirela Resende \\ Nassur ${ }^{3}$ \\ 1.Pesquisadora, Doutora em Ciência dos Alimentos, Embrapa Semiárido - Petrolina \\ (PE), Brasil. \\ 2. Graduanda, Tecnologia de Alimentos - Instituto Federal do Sertão Pernambucano - \\ Campus Petrolina - Petrolina/PE \\ 3. Docente, Doutora em Ciência dos Alimentos, Universidade do Estado da Bahia - \\ UNEB -Departamento de Tecnologia e Ciências Sociais - Juazeiro (BA), Brasil. \\ ritarnassur@hotmail.com
}

Recebido em: 06/04/2018 - Aprovado em: 10/06/2018 - Publicado em: 20/06/2018 DOI: 10.18677/EnciBio_2018A25

\begin{abstract}
RESUMO
O resgate de benefícios de compostos advindos de resíduos de alimentos tendem não somente a minimizar o descarte, como também ir de encontro à intensa demanda da população por alimentos com maiores benefícios à saúde. O Brasil está entre um dos maiores produtores, processadores e consumidores de mangas, em especial a região do Vale do São Francisco. Diante do exposto, o presente estudo avaliou a farinha de casca de manga das quatro principais variedades de manga cultivadas no Vale do São Francisco, 'Kent', 'Keitt', 'Palmer' e 'Tommy Atkins'. Frutos foram recebidos, selecionados quanto ao grau de maturação, higienizados e descascados manualmente, sendo a polpa utilizada para outros fins. Após secagem, trituração e peneiragem, as farinhas obtidas foram avaliadas em relação à umidade, fibras (FDN e FDA), lipídeos, minerais (cinzas), proteínas, carboidratos, ph, acidez, sólidos solúveis totais, cor, capacidade antioxidante e conteúdo de fenólicos totais. Farinhas da casca de mangas Palmer e Kent apresentaram cerca de $65 \%$ de sólidos solúveis e menores teores foram obtidos em análises para Keitt, condizendo com as maiores concentrações de fibras nesse último. As farinhas obtidas não são boas fontes proteicas, porém são fontes de compostos fenólicos e atividade antioxidante, o que pode aumentar teores de compostos nutricionais de interesse caso sejam adicionadas em formulações de novos produtos alimentícios, independente da variedade escolhida.
\end{abstract}

PALAVRAS-CHAVE: Atividade antioxidante, Fibras, Resíduo 


\title{
CHARACTERIZATION OF THE SKIN FLOUR FROM DIFFERENT MANGO CULTIVARS
}

\begin{abstract}
The recovery of benefits from compounds derived from food residues tend not only to minimize the fruit industry waste, but also to meet the population's intense demand for foods with higher health benefits. Brazil is one of the largest producers, processors and consumers of the fruit, especially the region of the São Francisco Valley. Thus, the present study evaluated the mango skin composition of the four main mango varieties cultivated in the San Francisco Valley: 'Kent', 'Keitt', 'Palmer' and 'Tommy Atkins'. Fruits were harvest, selected according to maturation, sanitized and manually peeled, being the flesh used for other purposes. After drying, crushing and sieving, the obtained flours were evaluated for moisture, fiber (NDF and ADF), lipids, minerals (ashes), proteins, carbohydrates, $\mathrm{pH}$, acidity, total soluble solids, color, antioxidant capacity and phenolic compounds. Palmer and Kent mango flour presented approximately $65 \%$ of soluble solids and lower levels were observed for Keitt, matching the higher fiber concentrations for the last one. The obtained flours are not good sources of protein but can act as sources of phenolic compounds and antioxidant activity, which can increase contents of nutritional compounds of interest if flours are added in formulations of new food products, regardless the mango variety.
\end{abstract}

KEYWORDS: Antioxidant activity, Fibers, Mangifera indica, Residue

\section{INTRODUÇÃO}

O Brasil está entre os maiores produtores e exportadores de manga a nível mundial. A manga é a segunda fruta mais exportada no país em termos de volume, e a primeira em termos de receita (SEBRAE, 2016). Os principais estados produtores de manga são: São Paulo, Bahia e Pernambuco, sendo a maior exportadora nacional, a região do Vale do São Francisco. A manga destaca-se como o fruto tropical mais popular do mundo, com recordes de produção de cerca de 30 milhões de toneladas (CHOON et al., 2018).

Muitos alimentos podem ser utilizados de forma integral, podendo ser uma ação de sustentabilidade e melhoria da qualidade alimentar, uma vez que grandes teores de nutrientes são encontrados em partes não convencionais (FRANZOSI et al., 2018). No entanto, pouco da fruta é utilizada para processamento, que aproveita excedentes de frutos e aumenta a vida útil dos mesmos. Além disso, após o processamento da manga, casca e semente são descartadas, gerando resíduos de 40 a $50 \%$ em massa da fruta (FAO 2009). A industrialização da casca pode ser uma alternativa para abrandar a geração de resíduos orgânicos sólidos e produzir alimentos saudáveis, pela incorporação de fibras e compostos com atividade antioxidante (DAMIANI et a., 2009).

Ultimamente, o resgate de benefícios de compostos bioativos advindos de resíduos de alimentos tendem não somente a minimizar o descarte, como também ir de encontro à intensa demanda da população por compostos fenólicos, os quais possuem efeitos protetivos contra doenças crônica (CHOON et al., 2018).

As fibras alimentares têm sido amplamente estudadas por suas propriedades funcionais. A Anvisa dispõe que alimentos funcionais, além de funções básicas, deve 
produzir efeitos metabólicos, fisiológicos, ou benéficos à saúde, devendo ser seguro sem supervisão médica. $E$ no caso de nutrientes com funções reconhecidas pela comunidade científica, não é necessária a demonstração de eficácia ou análise da mesma para alegação funcional (BRASIL, 1999).

A exploração de subprodutos do processamento como fonte de fibras e a aplicação em alimentos é um campo promissor, bem como a crescente vertente de alimentos com propriedades funcionais e que promovem benefícios à saúde humana. Aliada a esta demanda, verifica-se a importância da cultura da mangueira para o semiárido brasileiro e a necessidade de aproveitamento dos frutos que não são comercializados in natura. Desta forma, este trabalho pretendeu avaliar a farinha de casca de manga das quatro principais variedades de manga cultivadas no Vale do São Francisco, 'Kent', 'Keitt', 'Palmer' e 'Tommy Atkins'.

\section{MATERIAL E MÉTODOS}

Aproximadamente 30 quilos de mangas das variedades 'Kent', 'Keitt', 'Palmer' e 'Tommy Atkins' foram adquiridas do BAG da Embrapa Semiárido e de produtores locais. As mangas foram recebidas e armazenadas a $17^{\circ} \mathrm{C}$ para retirada do calor de campo. Os frutos foram selecionados de acordo com o grau de maturação, sendo utilizadas as mangas maduras para o experimento. Em seguida foram higienizadas com hipoclorito de sódio (15 ppm) por 20 minutos, bem como os utensílios utilizados no processo.

Cada variedade em separado foi então descascada manualmente, sendo a polpa utilizada para outros experimentos. As cascas foram secas em secador de fluxo de ar contínuo e ascendente em leito fixo (cabine) com sete bandejas de 0,25 $\mathrm{m}^{2}$ (SULAB), a $60^{\circ} \mathrm{C}$ por 24 horas. Após a secagem, foram trituradas em moinho de facas e então peneiradas em peneira de 60 mesh $(250 \mu \mathrm{m})$, ficando com granulometria semelhante à farinha de trigo comercial.

Para a determinação do $\mathrm{pH}$ e acidez total titulável (ATT) a farinha foi diluída em 1:11 (farinha/água destilada), sendo $1,0 \mathrm{~g}$ desta solução diluído em $50 \mathrm{~mL}$ de água destilada. Ambos resultados foram obtidos no equipamento Metrohn 848 Titrino Plus, com hidróxido de sódio $0,1 \mathrm{~N}$. O teor de sólidos solúveis foi obtido no refratômetro digital Pal-3, da Atago.

A umidade, lipídeos, cinzas e proteínas foram determinadas pelos métodos clássicos, segundo técnicas descritas pelo Instituto Adolfo Lutz (2005), e o teor de carboidratos por diferença. As fibras em detergente neutro (FDN) e fibras em detergente ácido (FDA), foram avaliadas pelo método de Van Soest (1967) e Goering e Van Soest (1970), respectivamente.

As análises de cor foram realizadas utilizando espectrofotômetro Delta Color, modelo delta Vista $450 \mathrm{G}$, com diagrama tridimensional de cores $\left(L^{*}, C^{*}, \mathrm{~h}\right)$, no qual $L^{*}$ indica a luminosidade, $C^{*}$ o Croma, indicando a saturação de cor, e h o ângulo de hue, ou tonalidade, valor em graus no diagrama tridimensional de cores: $0^{\circ}$ (vermelho), $90^{\circ}$ (amarelo)180 (verde) e $270^{\circ}$ (azul). A determinação da atividade antioxidante foi realizada pela captura de radicais livres a partir do método DPPH (RUFINO et al., 2010) e a avaliação do teor de fenólicos totais foi alcançada pelo método Folin-Ciocalteu, de acordo com Nuutila et al., (2003). Para todas as variáveis, as médias obtidas foram analisadas com auxílio do software SISVAR, com teste de médias para os fatores qualitativos. 


\section{RESULTADOS E DISCUSSÃO}

As farinhas de casca de manga apresentaram rendimento de cerca de $5 \%$ em relação ao peso da manga, sendo que a casca fresca representa cerca de $1 / 3$ do peso da fruta in natura. O rendimento médio das farinhas em relação ao peso inicial das cascas de manga foi de $15,72 \%$. Braga et al. (2013) obtiveram rendimento de farinha de $19,8 \%$ quando foi utilizada pelos autores uma peneira de 40 mesh. Para as análises da composição centesimal e fisíco-químicas, os resultados estão apresentados na Tabela 1.

TABELA 1: Resultados de composição e análises fisico-químicas para as farinhas das cascas de manga.

\begin{tabular}{|c|c|c|c|c|c|c|c|c|}
\hline Variedade & $\begin{array}{l}\text { Umidad } \\
\text { e (\%) }\end{array}$ & $\begin{array}{l}\text { Proteína } \\
\text { s (\%) }\end{array}$ & $\begin{array}{l}\text { Cinzas } \\
(\%)\end{array}$ & $\begin{array}{l}\text { Lipídeo } \\
\text { s (\%) }\end{array}$ & $\begin{array}{l}\text { Carboid } \\
\text { ratos } \\
\text { totais } \\
(\%)\end{array}$ & $\mathrm{pH}$ & $\begin{array}{l}\text { Acidez } \\
\text { (\%ác } \\
\text { cítrico) }\end{array}$ & $\begin{array}{l}\text { SS } \\
\left({ }^{\circ} \text { Brix }\right)\end{array}$ \\
\hline $\begin{array}{l}\text { Tommy } \\
\text { Atkins }\end{array}$ & $8,17^{a}$ & $0,82^{\mathrm{a}}$ & $3,13^{c}$ & $2,05^{b}$ & $85,83^{a}$ & $4,22^{\mathrm{a}}$ & $0,11^{\mathrm{c}}$ & $59,77^{b}$ \\
\hline Palmer & $8,30^{a}$ & $0,63^{b}$ & $3,68^{\mathrm{ab}}$ & $1,50^{d}$ & $85,89^{a}$ & $4,31^{a}$ & $0,14^{b}$ & $65,27^{\circ}$ \\
\hline Keitt & $6,90^{\mathrm{b}}$ & $0,40^{d}$ & $3,79^{a}$ & $2,13^{a}$ & $86,78^{a}$ & $3,70^{a}$ & $0,20^{\mathrm{a}}$ & $43,27^{\circ}$ \\
\hline Kent & $7,99^{a}$ & $0,52^{\mathrm{c}}$ & $3,50^{\mathrm{b}}$ & $1,61^{\mathrm{c}}$ & $86,38^{a}$ & $4,21^{a}$ & $0,19^{a}$ & $65,27^{a}$ \\
\hline CV\% & 2,72 & 3,93 & 2,72 & 5,11 & 2,00 & 6,66 & 2,55 & 3,03 \\
\hline
\end{tabular}

Valores com letras em comum em uma mesma coluna não diferem estatisticamente entre si segundo o teste de Tukey $(p \leq 0,05)$. SS: sólidos solúveis. $C V \%$ : coeficientes de variabilidade

De acordo com os teores de umidade (Tabela 1) verificou-se que os mesmos foram abaixo do valor máximo estabelecido pela legislação vigente para farinhas vegetais (BRASIL, 1978), e não houve diferença estatística entre as variedades. Ao desenvolverem doces com cascas de banana, Claudy et al. (2014) obtiveram umidades de $38,84 \%$, valor justificado pela natureza diferenciada dos produtos de resíduos de frutas. Também não houve diferença estatística para o $\mathrm{pH}$.

A acidez total titulável foi maior para Keitt e Kent (Tabela 1), no entanto com valores muito próximos da Palmer e Tommy Atkins. Braga et al., (2013) encontraram pH 4,54 e acidez titulável de $0,94 \mathrm{~g}$ (\% ácido cítrico) para farinha da casca de manga 'Tommy Atkins', com valores similares de pH e maior acidez em relação ao presente estudo.

A variedade que apresentou o maior teor de proteínas foi a Tommy Atkins $(0,82 \%)$, no entanto, como esperado, os valores são bastante baixos, não sendo considerados fontes protéicas. O componente que se destaca nas cascas são os carboidratos totais, não havendo diferença estatística entre as variedades, e estando todos acima de $85,8 \%$. A quantidade de sólidos solúveis foi igualmente alta nas amostras, havendo bastante discrepância para a Keitt, que apresentou apenas 43,27 'Brix (Tabela 1). O teor de carboidratos totais é diretamente influenciado pelo alto teor de fibras, como é apresentado na Tabela 2.

TABELA 2: Resultados de Fibra Detergente Ácido (FDA) e 
Fibra Detergente Neutro (FDN) para as farinhas das cascas de manga.

\begin{tabular}{lrr}
\hline Variedade de manga & FDA (\%) & FDN (\%) \\
\hline Tommy Atkins & $19,33^{\mathrm{a}}$ & $21,15^{\mathrm{b}}$ \\
Palmer & $16,44^{\mathrm{b}}$ & $21,69^{\mathrm{ab}}$ \\
Keitt & $14,05^{\mathrm{c}}$ & $22,71^{\mathrm{a}}$ \\
Kent & $12,9^{\mathrm{d}}$ & $17,17^{\mathrm{c}}$ \\
CV\% & 2,52 & 2,45 \\
\hline
\end{tabular}

Produtos com letras em comum em uma mesma coluna não diferem estatisticamente entre si segundo o teste de Tukey $(p \leq 0,05)$. CV\%: coeficientes de variabilidade

Para a análise de fibras (Tabela 2) foram encontradas diferenças estatísticas entre as amostras, sendo o maior teor encontrado para a manga Keitt $(22,71 \%)$, o que pode explicar o menor teor de sólidos solúveis presentes, apesar do teor de carboidratos totais similar ao de outras variedades. Fibra em detergente neutro (FDN) e Fibra em detergente ácido (FDA) referem-se às porções pouco digeríveis da parede celular, sendo que a fibra em detergente ácido (FDA) é constituída, basicamente, de lignocelulose, ou seja, lignina mais celulose enquanto a fibra em detergente neutro (FDN) compreende frações de celulose, hemicelulose e lignina. A diferença entre essas duas frações (FDA e FDN) pode ser considerada uma estimativa do teor de hemicelulose na amostra (CECCHI, 2007).

Os valores obtidos neste estudo são comparativamente baixos, porém, quando a metodologia de obtenção da farinha é avaliada, diferenças na granulometria de materiais avaliados são obtidas. Quando uma farinha com granulometria similar à da farinha de trigo é obtida, a probabilidade de percepção do consumidor à sua adição é reduzida, com a possibilidade de aumento de fontes em que a mesma pode ser adicionada, como sucos, doces e etc. Em geral, farinhas com maiores granulometrias contem maior teor de fibras, com aumento da probabilidade de rejeição por consumidores, sendo indicada para adição em bolos, pães, etc.

Damiani et al., (2009) apresentaram 15,80\% de fibra insolúvel em casca de manga cv. Haden. Foi encontrado $37,10 \%$ de fibra alimentar na farinha da casca de manga 'Tommy Atkins' (BRAGA et al., 2013). Marques et al., (2010) afirmam que $100 \mathrm{~g}$ de casca de manga 'Tommy Atkins' corresponde a $50 \%$ do consumo diário de fibras recomendado por pessoa e contribuem na prevenção de doenças cardiovasculares e redução dos níveis séricos de glicose e lipídeos.

No entanto, há discrepância entre teores de diferentes materiais e estudos, proveniente devido à aplicação de diferentes métodos, com resultados possíveis em Fibra Detergente ácido (FDA), Fibra Detergente Neutro (FDN), Fibra Bruta e Fibra Alimentar. Há grande evidência científica que demonstra a presença de compostos bioativos em diferentes cascas de frutos (CHOON et al., 2018). Uma fração rica em 
fibra solúvel da casca de abacaxis resultou em potencial em melhorar funções intestinais in vivo (HUANG et al., 2014).

TABELA 3: Coloração, capacidade antioxidante e fenólicos totais em farinhas das cascas de manga.

\begin{tabular}{|c|c|c|c|c|c|}
\hline Variedade & $L^{*}$ & $C^{*}$ & h & $\begin{array}{l}\text { Capacidade } \\
\text { Antioxidante } \\
\text { (\%SRL) }\end{array}$ & $\begin{array}{l}\text { Fenólicos } \\
\text { (ug/mg) } \\
\text { EAG }\end{array}$ \\
\hline Tommy Atkins & $70,75^{b}$ & $34,78^{c}$ & $83,26^{b}$ & $89,29^{a}$ & $902,65^{\mathrm{ab}}$ \\
\hline Palmer & $67,56^{c}$ & $39,55^{b}$ & $82,51^{b c}$ & $90,27^{a}$ & $819,71^{b}$ \\
\hline Keitt & $74,15^{\mathrm{a}}$ & $31,48^{d}$ & $85,50^{a}$ & $90,89^{a}$ & $1042,40^{a}$ \\
\hline Kent & $70,26^{b}$ & $41,92^{a}$ & $81,76^{c}$ & $91,43^{a}$ & $912,91^{\mathrm{ab}}$ \\
\hline CV\% & 0,78 & 1,08 & 0,40 & 1,09 & 7,28 \\
\hline
\end{tabular}

$L^{*}$ : luminosidade, $C^{*}$ : Croma, indicando a saturação de cor, e h: ângulo de hue, ou tonalidade, valor em graus no diagrama tridimensional de cores: $0^{\circ}$ (vermelho), $90^{\circ}$ (amarelo), 180 (verde) e 270 (azul). Produtos com letras em comum em uma mesma coluna não diferem estatisticamente entre si segundo o teste de Tukey $(p \leq 0,05)$. CV\%: coeficientes de variabilidade.

O resultado da análise de cor realizado em todas as farinhas foi próximo à tonalidade amarela, sendo a Keitt $(h=85,50)$ a mais amarela e a Kent a menos amarela $(\mathrm{h}=81,76)$. Todas as amostras apresentaram alta luminosidade, sendo a menos luminosa (mais escura) a farinha da casca de Palmer $\left(L^{*}=67,56\right)$. A farinha da casca de Keitt além de ser a mais amarela também é a mais clara, no entanto apresentou menor saturação de cor $\left(C^{*}=31,48\right)$. Cascas de mangas apresentaram alta capacidade antioxidante (cerca de $90 \%$ de sequestro de radicais livres), independente da cultivar estudada, as quais não diferiram significativamente entre si, de acordo com o teste de Tukey.

Farinhas obtidas de cascas de mangas Palmer resultaram em menores teores (22\% menor) de compostos fenólicos quando comparadas com o mesmo produto obtido de manda Keitt, variação que pode ser atribuída à fatores fisiológicos e genéticos intrínsecos do material vegetal, que foram produzidos na mesma área experimental. Os demais materiais avaliados encontram-se em proporções intermediárias em $\mathrm{ug} / \mathrm{mg}$ de equivalente de ácido gálico (EAG). Os resultados de obtidos no presente estudo são altos quando comparados com teores de farinhas de

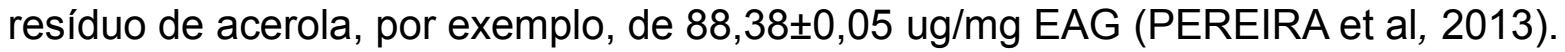

Grande quantidade de compostos metabólitos podem ser encontrados em cascas de várias frutas e hortaliças e suas funções estão associadas com conteúdo de compostos fenólicos nesses alimentos (MARTINEZ-AVILA et al., 2014), que resulta em menor incidência de doenças degenerativas e diminuição de fatores de risco das mesmas. Além disso, compostos fenólicos tem sido associados a efeitos mutagênicos e boa atividade antioxidante (MARTINEZ-AVILA et al., 2014). Hernandez-Santos et al. (2014) também observaram efeitos antioxidantes quando estudaram a casca despectinizada de maracujás.

Ratos suplementados com dietas com cascas de manga em níveis de 5 e $10 \%$ demonstraram aumento no açúcar da urina, volume de urina e aumento de 
lipoproteínas de alta densidade (HDL), o que implica que a casca da manga pode ser adotada como um ingrediente funcional com efeito antidiabético (GONDI et al., 2015).

Além dos parâmetros de interesse nutricional e bioativos no presente estudo avaliados, foram reportados altos teores de minerais na casa e semente de mangas (CHIOCCHETTI et al., 2013), acima de valores da polpa, assim como Fasoli e Righetti (2013) relataram cerca de 334 proteínas diferentes na casca do fruto citado. Componentes bioativos foram também encontrados, além da casca de mangas (LUO et al., 2014), em extratos de casca de rambutan (LESTARI et al., 2014), pitaia (LIAOTRAKOON et al., 2013), jaca (MADRUGA et al., 2014) e maracujá (LEWIS et al., 2013).

\section{CONCLUSÕES}

Farinhas da casca de manga das variedades apresentadas surgem como uma opção para incremento de renda, com comprovado benefício nutricional e tecnológico, podendo ser passíveis de utilização em produtos do processamento do próprio fruto ou a serem adicionados em outros alimentos visando a incrementação de fibras ou da atividade antioxidante do produto.

\section{REFERÊNCIAS}

BRAGA, B. A.; CARVALHO, S. A.; SOUZA, A. O. Avaliação físico-química e da qualidade microbiológica de farinhas produzidas a partir de resíduos agroindustriais. Higiene Alimentar. $\quad$ v. 27, p. 3871-3875, 2013. Disponível em: < http://www2.uesb.br/ppg/ppgecal/wp-content/uploads/2017/04/BRUNA-BRAGA.pdf > .

BRASIL. Decreto $n^{\circ} \mathbf{1 2 . 4 8 6}$, de 20 de outubro de 1978. Normas técnicas especiais relativas a alimentos e bebidas. Diário Oficial do Estado de São Paulo, São Paulo, p. 20, 21 out. 1978.

BRASIL. Ministério da Saúde. Agência Nacional de Vigilância Sanitária. Resolução n. 18 , de 30 de abril de 1999. Aprova o Regulamento Técnico que Estabelece as Diretrizes Básicas para Análise e Comprovação de Propriedades Funcionais e ou de Saúde Alegadas em Rotulagem de Alimentos. Brasília, 1999.

$\mathrm{CECCHI}, \mathrm{H}$. M. Fundamentos teóricos e práticos em análise de alimentos. Campinas: Editora da Unicamp, 2007.

CHIOCCHETTI, G.M.; NADAI FERNANDEZ, E.A.; BACCHI, M.A.; PAZIM, R.A.; SERRIÉS, S.R.V.; TOMÉ, T.M. Mineral composition of fruit by-products evaluated by neutron activation analysis. Journal of Radioanalytical and Nuclear Chemistry. v. 297, n.3, p. 399-404, 2013. Disponível em: < https://link.springer.com/article/10.1007/s10967-012-2392-8>. Doi: 10.1007/s10967012-2392-8

CHOON Y. CHEOK, C.Y.; ADZAHAN, N. M.; RAHMAN, R. A.; ABEDIN, N. H. Z.; HUSSAIN, H.; SULAIMAN R.; CHONG, G. H. Current trends of tropical fruit waste utilization. Critical Reviews in Food Science and Nutrition. V.58, n.3, p. 335-361. 2018. Disponível em:< http://dx.doi.org/10.1080/10408398.2016.1176009>. Doi: 
CLAUDY, L.; SERBAI, D.; SANTOS, E. F.; MANHANI, M. R.; SILVA, É. C.; NOVELLO, D. Brigadeiro adicionado de aveia e banana: caracterização físicoquímica e sensorial entre crianças. Evidência, v.14, n.1, p.35-46, 2014. Disponível em: < http://editora.unoesc.edu.br/index.php/evidencia/article/view/4175/pdf_10>

DAMIANI, C.; VILAS BOAS E. V. B.; SOARES JUNIOR M. S., CALIARI M., PAULA M. L., ASQUIERI E. R. Avaliação química de geleias de manga formuladas com diferentes níveis de cascas em substituição à polpa. Ciência e Agrotecnologia. v.33 n.1, p.177-184, 2009. Disponível em: < http://www.scielo.br/scielo.php? script=sci arttext\&pid=S1413-70542009000100025>. Doi: $\quad$ 10.1590/S141370542009000100025

FASOLI, E.; RIGHETTI, P. G. The peel and pulp of mango fruit: A proteomic samba. Biochimica et Biophysica Acta. v.1834, n.12 , p.2539-2545, 2013. Disponível em: <https://www.ncbi.nlm.nih.gov/pubmed/24056186.> 10.1016/j.bbapap.2013.09.004

FOOD AND AGRICULTURE ORGANIZATION OF THE UNITED NATIONS (FAO). A manga no mundo e no Brasil. 2009. Disponível em: <https://www.fao.org.br>. Acesso em: 10 mar. 2017.

FRNZOSI, D.; DANELUZ, H. C.; BARATTO, I. Níveis de desperdício de produtos utilizados diariamente em um restaurante no sudoeste do Paraná. Revista Brasileira de Obesidade, Nutrição e Emagrecimento. v.12, n.69, p. 66-75, 2018. Disponível em: < https://dialnet.unirioja.es/servlet/articulo?codigo=6301523>

GOERING, H. K.; VAN SOEST, P. J. Forage fiber analysis: apparatus, reagents, procedures and some applications. Washington, DC: USDA, 1970. (Agriculture handbook, n. 379).

GONDI, M.; BASHA, S. A.; BHASKAR, J. J.; SALIMATH, P. V.; RAO, U. J. Antidiabetic effect of dietary mango (Mangifera indica L.) peel in streptozotocin-induced diabetic rats. Journal of the Science of Food and Agriculture. v.95, n.5, p.991999, 2015. Disponível em: < https://www.ncbi.nlm.nih.gov/pubmed/24917522> doi: 10.1002/jsfa.6778. Epub 2014 Jul 10.

HERNÁNDEZ-SANTOS, B.; VIVAR-VERA, M. A.; RODRÍGUEZ-MIRANDA, J.; HERMAN-LARA, E.; TORRUCO-UCO, J.G.; ACEVEDO-VENDRELL, O. Dietary fibre and antioxidant compounds in passion fruit (Passiflora edulis f. flavicarpa) peel and depectinised peel waste. International Journal of Food Science and Technology. V. 50, n.1, p.268-274, 2014. Disponível em: < https://onlinelibrary.wiley.com/doi/abs/10.1111/ijfs.12647> doi: doi.org/10.1111/ijfs.12647

HUANG, Y.L.; TSAI, Y. H.; CHOW, C. J. Water-insoluble fiber-rich fraction from pineapple peel improves intestinal function in hamsters: evidence from cecal and fecal indicators. Nutrition Research. v. 34, n.4, p.346-354, 2014. Disponível em: < ENCICLOPÉDIA BIOSFERA, Centro Científico Conhecer - Goiânia, v.15 n.27; p. 19 
https://www.ncbi.nlm.nih.gov/pubmed/24774071>. Doi: 10.1016/j.nutres.2014.03.001.

INSTITUTO ADOLFO LUTZ (IAL). Normas analíticas do Instituto Adolfo Lutz. Métodos físico-químicos para análise de alimentos. 4. ed. Brasília: Ministério da Saúde, Agência Nacional de Vigilância Sanitária, 2005. 1018 p. (Série A - Normas e Manuais Técnicos).

LESTARI, S.R.; DJATI, M.S.; RUDIJANTO, A.; FATCHIYAH, F. The physiological response of obese rat model with rambutan peel extract treatment. Asian Pacific Journal of Tropical Disease. v. 4, n.2, p.780-785, 2014. Disponível em:< https://www.sciencedirect.com/science/article/pii/S222218081460726X> doi: 10.1016/S2222-1808(14)60726-X

LEWIS, B.J.; HERRLINGER, K.A.; CRAIG, T.A.; MEHRING-FRANKLIN, C.E.; DEFREITAS, Z.; HINOJOSA-LABORDE, C. Antihypertensive effect of passion fruit peel extract and its major bioactive components following acute supplementation in spontaneously hypertensive rats. The Journal of Nutritional Biochemistry. v. 24, n.7, p1359-1366, 2013. Disponível em: https://www.ncbi.nlm.nih.gov/pubmed/23333089>. Doi: 10.1016/j.jnutbio.2012.11.003

LIAOTRAKOON, W.; BUGGENHOUT, S.V.; CHRISTIAENS, S.; HOUBEN, K.; CLERCQ, N.; DEWETTINCK, K. An explorative study on the cell wall polysaccharides in the pulp and peel of dragon fruits (Hylocereus spp.). European Food Research and Technology. v. 237, n.3, p.341-351, 2013. Disponível em: < https://link.springer.com/article/10.1007/s00217-013-1997-7> doi: 10.1007/s00217013-1997-7.

LUO, F.; FU, Y.; XIANG, Y.; YAN, S.; HU, G.; HUANG, X. Identification and quantification of gallotannins in mango (Mangifera indica L.) kernel and peel and their antiproliferative activities. Journal of Functional Foods. v.8, n.1, p.282-291, 2014. Disponível em: <http://agris.fao.org/agris-search/search.do? recordID=US201700165799> doi: 10.1016/j.jff.2014.03.030

MADRUGA, M.S.; ALBUQUERQUE, F.S.M.; SILVA, I.R.A.; AMARAL, D.S.; MAGNANI, M.; NETO, V.Q. Chemical, morphological and functional properties of Brazilian jackfruit (Artocarpus heterophyllus L.) seeds starch. Food Chemistry. v.143, p. 440-445, 2014. Disponível em: < https://www.ncbi.nlm.nih.gov/pubmed/24054264> doi: 10.1016/j.foodchem.2013.08.003

MARQUES, A.; YABAN G. C.; ARAÚJO M. T.; MANHAES L. R. T.; SABAA-SRUR A. U. O. Composição Centesimal e de Minerais de Casca e polpa de Manga (Mangifera indica L.) cv. Tommy Atkins. Revista Brasileira de Fruticultura. v. 32, n. 4, p. 12061210, 2010. Disponível em: <http://www.scielo.br/scielo.php? script=sci_arttext\&pid=S0100-29452010000400031> doi: 10.1590/S010029452010005000117

MARTINEZ-AVILA, G. C. G.; AGUILERA, A.F.; SAUCEDO, S.; ROJAS, R.; 
RODRIGUEZ, R.; AGIULAR C.N. Fruit wates fermentation for phenolic antioxidants production nad their application in manufacture of edible coatings and films. Critical Reviews in Food Science and Nutrition. v.54, n.3, p.303-311, 2014. Disponível em: <https://www.tandfonline.com/doi/abs/10.1080/10408398.2011.584135> doi: 10.1080/10408398.2011.584135

NUUTILA, A. M.; PUUPPONEN-PIMIÄ, R.; AARNI, M.; OKSMAN-CALDENTEY, K. Comparison of antioxidant activities of onion and garlic extracts by inhibition of lipid peroxidation and radical scavenging activity. Food Chemistry, v.81, p.485-493, 2003.

Disponível em:

https://www.sciencedirect.com/science/article/pii/S0308814602004764> doi: 10.1016/S0308-8146(02)00476-4.

PEREIRA, C.T.M., SILVA, C.R.P., LIMA, A., PEREIRA, D.M., COST, C.N., NETO, A.A.C. Obtenção, caracterização físico-química e avaliação da capacidade antioxidante in vitro da farinha de resíduo de acerola (Malpighia glabra L.). Acta Tecnológica, v.8, n.2, p. 50-56, 2013. Disponível em: < http://portaldeperiodicos.ifma.edu.br/index.php/actatecnologica/article/view/213>

RUFINO, M. S. M.; ALVES, R. E.; BRITO, E. S.; JIMÉNEZ. J. P.; CALIXTO. F. S. Bioactive compounds and antioxidant capacities of 18 non-traditional tropical fruits from Brazil. Food Chemistry, v. 121, n. 4, p. 996 - 1002, 2010. Disponível em: < https://www.sciencedirect.com/science/article/pii/S0308814610001172> doi: https://doi.org/10.1016/j.foodchem.2010.01.037

SEBRAE. Fruticultura - Cenários e projeções estratégicas. 30p., 2016. Disponível em <http://www.bibliotecas.sebrae.com.br/chronus/ARQUIVOS_CHRONUS/bds/bds.nsf/ e93e6e44c0b1ec9bed5f9ed186ab6b7e/\$File/6083.pdf>

VAN SOEST, P. J. Development of a comprehensive system of feed analysis and its application to forages. Journal of Animal Science, v.26, p.119-128, 1967. Disponível em: < https://academic.oup.com/jas/article/26/1/119/4698238>. Doi: 10.2527/jas1967.261119x 